Trabajos y Comunicaciones, 2da. Época, No 46, e044, octubre 2017. ISSN 2346-8971

Universidad Nacional de La Plata.

Facultad de Humanidades y Ciencias de la Educación.

Departamento de Historia

\title{
Clase, capitalismo, y contingencia nacional en la obra de René Zavaleta
}

\section{Class, Capitalism, and National Contingency in the Works of René Zavaleta}

\section{Robert Cavooris*}

* History of Consciousness Department, University of California, Santa Cruz, Estados Unidos | rcavooris@gmail.com

\section{PALABRAS CLAVE}

Nación

Composición de clase

Sujeto político

Zavaleta

Abigarramiento

\section{KEYWORDS}

Nation

Class composition

Political subject

Zavaleta

Motley society

\section{RESUMEN}

René Zavaleta persiguió la relación de la forma nación y la lucha de clases a lo largo de su vida, buscando siempre vincularla a la cuestión de un sujeto político colectivo. Este ensayo traza su manera cambiante de relacionar estos conceptos desde su fase nacionalista revolucionaria hasta sus reflexiones maduras en Lo nacional-popular en Bolivia. Se plantea que por una transformación política y epistemológica, Zavaleta llegó a pensar clase y nación en conexión con las tendencias de descomposición y composición en el capitalismo, desarrollando así una visión de la nación como históricamente contingente que hoy puede utilizarse para analizar el neoliberalismo.

\section{ABSTRACT}

René Zavaleta investigated the relationship between class struggle and the nation form throughout his life, seeking to link it to the question of the collective political subject. This essay traces his shifting way of relating these concepts from his revolutionary nationalist phase through his mature reflections in Lo nacional-popular en Bolivia. I argue that through a political and epistemological transformation, Zavaleta came to think class and nation in their connection to the tendencies of composition and decomposition within capitalismo, thus developing a view of the nation as historically contingent which might be used to analyze neoliberalism today. 


\section{Introducción: La teoría, la historia, y la cuestión del sujeto político}

La teor ía política es siempre construida sobre una fisura interna de sus propios términos. Aunque aspira a lo universal, lo generalizable, y lo abstracto, lleva siempre la carga de la política, es decir un compromiso a investigar el poder existente y las luchas del mundo que la marcan indeleblemente. Pocos han vivido esta tensión más plenamente que René Zavaleta Mercado, para quien la cuestión de la producción del conocimiento teórico es inseparable de la posición histórica del productor, y por tanto siempre atada a eventos políticos concretos. "Uno conoce, como es natural, desde lo que es,” escribe (Zavaleta, 2013b: 643). Esto no es decir que por eso el conocimiento nazca con límites a priori debidos a la identidad reconocida de su productor, o que sea en algún sentido no transmisible fuera de su contexto original. Al contrario, el conocimiento producido en circunstancias históricas concretas no puede ser estático porque la historia misma tampoco lo es. Momentos de gran cambio abren la posibilidad de nuevos encuentros entre ideas y contextos, conceptos y eventos. Como escribe Luis Tapia (2002):

"La intensificación del tiempo histórico que se produce en y con una revolución es un poderoso acicate que activa el trabajo intelectual e historiográfico. Dicho de otro modo, la intensificación del tiempo histórico pide que se lo interprete, que se lo reconstruya desde el pasado, que se lo reflexione, y también que se lo dirija” (p. 146).

Así eran las tareas descubiertas por Zavaleta con su fidelidad a la revolución nacional boliviana de 1952, las cuales abrieron la relación inestable entre la teoría y la política.

Esta relación históricamente cambiante también empuja la cuestión de la producción del conocimiento hacia otra, la del sujeto político. El teórico reacciona frente la historia, pero ¿̇a quién está destinado el conocimiento producido? ¿Qué tipo de sujeto se constituye en la creación y recepción de las ideas, allí en el espacio entre la potencia infinita del pensamiento y la realidad aparentemente finita de sus efectos y fuentes? ¿Desde qué posición se entiende la política en la teoría política?

En las obras de su primera etapa, dentro de lo que normalmente se designa como su fase nacionalista, el horizonte subjetivo de Zavaleta fue el de la nación boliviana. Su preocupación fue explicar la especificidad de Bolivia, y en particular, pensar un proyecto nacional que pudiera dar sustancia real a su existencia como comunidad política y sujeto a nivel mundial. Sin embargo, las tareas intelectuales de Zavaleta crecieron desde y más allá de la coyuntura de 1952; él no podía evitar las condiciones vacilantes que surgieron de aquel momento, las que afectarían sin cesar su ser y su pensamiento. Mientras la revolución misma encontró sus límites, el asunto del sujeto político y su relación con el teórico tenía que reabrirse una y otra vez.

El resultado fue, a lo largo de la vida de Zavaleta, un cambio de dimensión tan epistemológico como político. Por el lado epistemológico, Zavaleta tuvo que profundizar su entendimiento no solo de la idea de la nación, sino de su falta -o sea, investigar la existencia de condiciones que obstruían la formación de algo que pudiera cumplir con una visión idealista de la nación boliviana como comunidad política. Para la tarea de analizar tal falta o carencia, una teoría general de la nación no era suficiente, pero tampoco servía ninguna investigación meramente empírica si no podía conectar las condiciones locales con la construcción teórica. Así Luis Antezana (1991) interpreta el comentario epigramático de Zavaleta (2013a): "Se sabe que la anécdota es la elocuencia de los hechos, pero también su encierro.” Por el lado político, las limitaciones de la revolución de 1952 y el reconocimiento por Zavaleta de las contradicciones de clase en el seno del proyecto nacional también le orientaron hacia un análisis más profundo de la lucha de clases, enfocándose en la importancia del aparato estatal y las ideologías de la clase dominante, además de las relaciones entre obreros y campesinos, sus historias, y sus posiciones estructurales cambiantes. Aun en la deseada construcción de un sujeto nacional, meta que Zavaleta nunca totalmente abandonó, la cuestión de un sujeto de clase no podía evitarse.

El objetivo del presente ensayo es seguir ciertos componentes de este cambio concurrentemente epistemológico y político en la obra de Zavaleta. Al intentar esto, veremos no solo que la relación entre el concepto de la nación y el 
de la clase se modificó, sino que los contenidos mismos de cada concepto cambiaron dramáticamente. Si seguimos esta tendencia en sus trabajos a sus conclusiones lógicas, encontraremos en Zavaleta una constelación conceptual que desplaza la nación como objeto central de investigación y deconstruye sus premisas, abriendo en su lugar los términos de la composición de clase y una noción contingente de la constitución de la unidad política. Prestar atención al cambio en el sitio conceptual del sujeto político y su relación con las posibilidades del conocimiento nos permitirá mejor captar, si no resolver, la tensión entre lo político y local por un lado y lo teórico por otro en los escritos de Zavaleta.

Sobre esta base de entendimiento, sugeriré que la utilidad que las producciones intelectuales de Zavaleta no pueden confinarse a la examinación de Bolivia, ni siquiera a Latinoamérica, sino que pueden servir para investigar mejor las dinámicas del capitalismo en todas sus articulaciones contemporáneas. En un mundo donde los contornos del imperialismo y del capitalismo han sido profundamente reformados por la transformación neoliberal, la posibilidad de pensar lo nacional, en la manera de Zavaleta, es muy distinta. También las composiciones de las clases se han modificado a nivel granular. Sin embargo, ciertos proyectos nacionalistas, aunque muy distintos, se presentan hoy como reacciones políticas al neoliberalismo tanto en América Latina como en Norteamérica y Europa. Por lo tanto, tenemos que preguntarnos sobre las coordenadas de cualquier proyecto nacional a la luz del mundo contemporáneo cambiante. Al refinar y retomar las trayectorias de Zavaleta en torno a esta cuestión, podemos ganar una herramienta teórica imprescindible para analizar nuestras coyunturas.

\section{EI nacionalismo revolucionario}

El joven Zavaleta fue bastante claro con respecto a su fidelidad a los eventos de Abril de 1952 en Bolivia, cuando un intentado putsch del Movimiento Nacionalista Revolucionario (MNR) fue apoyado en las calles por un levantamiento de obreros que exitosamente depuso al impopular gobierno conservador. Una década después, Zavaleta cuenta su experiencia formativa de ver los mineros armados, héroes de la revolución, bajando los cerros a la ciudad de Oruro para luchar contra los soldados fieles al régimen: “Así, supimos que cada hombre es en cierta medida del tamaño de su país y que la nacionalidad es un elemento del yo, que el yo individual no se realiza sino a través del yo nacional” (Zavaleta, 2011d: 536). En términos intelectuales, se ubica dentro de la tradición de nacionalismo revolucionario que precedió y en parte creó esa revolución, la cual ha James Dunkerley (2007) caracteriza como “la poética de una colectividad frustrada” (p. 227).

Para los intelectuales de esa tradición como Carlos Montenegro y Augusto Céspedes, quienes influyeron a Zavaleta, la historia de Bolivia fue una historia de los impedimentos a su cohesión auténtica y orgánica. Según Montenegro escribiendo en 1944, los eventos importantes desde la independencia "solo marcaron otro episodio más de la vieja lucha entre el coloniaje y la nacionalidad” (Montenegro, 1967: 98). Sin embargo, más allá de su anticolonialismo, esta problemática en el pensamiento político estuvo cargada con ambigüedades significativas, como el hecho de que sus exponentes más visibles expresaron afinidades hacia el fascismo italiano y alemán (Mansilla, 2015: 115-116). Así podemos colocar el nacionalismo boliviano de ese entonces dentro de la lucha global, a lo largo del siglo XX, sobre los términos de la acción política colectiva marcada por el comunismo y el fascismo, dos posiciones que en sus discursos siempre hicieron referencia a un cuerpo objetivo - o la clase, o la nación mítica (Badiou, 2007). Pero lo que caracteriza el referente en el caso de los nacionalistas Bolivianos no es sólo una historia mítica, sino su estatus siempre potencial, con la nación entendida como posibilidad histórica que debía afirmarse en la práctica por un proyecto histórico que vencería los impedimentos externos a la realización.

Por lo tanto, Montenegro (1967) busca en el pasado "lo nacional como energía histórica afirmativa y, por lo mismo, creadora y perpetuadora” (p. 25). Este entendimiento casi vitalista de la potencia de la nación aparece sintomáticamente a lo largo del análisis de Montenegro, y le permite la mediación de una relación contradictoria y aún aporética entre algo del porvenir, la nación como proyecto normativo, y algo ya existente operando bajo el nombre de Bolivia que tendía hacia ese futuro. Sirve, en otras palabras, para suturar la temporalidad irregular de la nación histórica. Se ve claramente esta concepción cuando, por ejemplo, él describe cómo el Mariscal Andrés de 
Santa Cruz rompió la dominación oligárquico-colonial en los años 1830: "El brazo del Mariscal conmovió como un cable eléctrico el cuerpo de la República. La tensión acumulada en el alma de aquel vástago de monarcas indios trasmitióse vibrante y continua por el espacio de diez años, a la nación. Él la encontró todavía intacta en su potencial de vida....” (Montenegro, 1967: 92). Así la nación considerada como potencia nunca disminuye, solo se hace aparente o no en algún momento según sus circunstancias. Zavaleta también explicita esta idea en 1957:

"La nacionalidad se mantiene no en las leyes ni en la organización territorial y sus normas meramente accesorias, sino en el pueblo. Pero el pueblo a su vez no puede realizar su destino histórico sino es a través de la independencia y la autonomía, es decir, la realización de la nación [noción potencial]" (2011b:532; cursivas añadidas).

Al final, es una concepción idealista, donde la forma de la historia es pre-definida como nacional, y lo nacional se piensa siempre como algo internamente coherente. Como observa Tapia (2002), lo imposible de pensar dentro de tales tipos de formulaciones es que "la nación misma pueda engendrar o contener el desarrollo y organización de sujetos y estructuras de su imposibilidad final” (p. 45).

Si la noción de la potencia intenta manejar una de las complicaciones de la interpretación nacionalista de la historia, la cuestión de la clase, el otro gran referente objetivo de la acción colectiva en el siglo XX, abre otra dificultad. Montenegro (1962), por su parte, reconoce que un análisis en los términos de nacionalismo y coloniaje no puede desconocer los conflictos de clase como "resorte propulsor del devenir boliviano," y él nota la importancia de los momentos en que se encuentran "todas las clases transitoriamente fusionadas por el descontento" contra el poder dominador (pp. 50, 75). No obstante, el uso de la categoría de clase en su discurso se articula siempre con el marco interioridad/exterioridad de la nación misma. Las clases aparecen como órganos coherentes en sí del cuerpo nacional cuya identidad se garantiza por la oposición a un poder colonizador. El problema importante para Montenegro entonces es entender cuáles clases serían capaces de actualizar la potencia nacional en el proceso de la formación del estado independiente. En otros términos, la nación sería la base objetiva de la política, pero se reconoce que las clases actúan como sujetos en su nombre.

Zavaleta retoma esta problemática cuando escribe sobre las dificultades del régimen de la revolución nacional continuante. En un texto escrito en 1962, explica que el MNR en su forma de 1952 había sido creado por un “encuentro de intereses" entre el proletariado, el campesinado, y las clases medias. Este método había sido posible porque, a nivel global, había una contradicción histórica entre los "explotadores y explotados,” y Bolivia como "nacionalidad explotada”, por eso se encontró en condiciones en las que las varias clases nacionales podían juntarse. Pero "a partir de 1952, se arrasa virtualmente con el poder rosquero [la oligarquía minera] y entonces aparecen nuevas y nuevas contradicciones que se explican a partir de la tesis del poder dual o doble poder" (Zavaleta, 2011d: 537-38). Se refiere a una lucha interna entre la Central Obrera Boliviana, la federación sindical del país, y el gobierno que quedó en manos de elementos de clase media. En este contexto, Zavaleta se ubica claramente del lado de los obreros y el socialismo, y es elegido como diputado izquierdista del MNR postulando que la única salida a la creciente crisis económica es dedicarse al desarrollo industrial estatalista y a expandir el proletariado más allá de la industria minera. Cualquiera intento de competir en términos capitalista, sostiene, sólo terminará con la explotación continuada del país entero. Lo notable de este argumento es el desplazamiento de la lucha de clases desde la esfera nacional a la internacional:

"La explotación imperialista se realizaba a través del Superestado [Boliviano] y se cumple ahora por el dominio del mercado de nuestros minerales. En consecuencia, los intereses del proletariado que luchaba contra el Superestado minero, que pertenecía al núcleo del imperialismo, eran también los intereses de la Nación y por eso el proletariado no era solamente una clase oprimida sino también una clase nacional y así la lucha de clases, que en principio parece un fenómeno relativo sólo a una sociedad determinada y aparte, se convierte en una lucha internacional entre las naciones proletarias o naciones marginales y las naciones opresoras. Por eso el nacionalismo de la Revolución Boliviana es legítimo sólo cuando se funda en la lucha de clases” (Zavaleta, 2011d: 541). 
El proletariado se concibe aquí como sujeto político con sus propios intereses estructurales. A la vez, es un sujeto cuyo referente objetivo sigue siendo la nación, concebida también con sus propios intereses, pero vista como algo efectivamente inerte debido a su posición a nivel mundial. Es decir, según Zavaleta lo que legitima el nacionalismo boliviano es la lucha de clases, pero la lucha de clases en Bolivia se legitima sólo por la lucha de clases internacional expresada por naciones explotadas y explotadoras. Es una posición teórica bastante complicada, tal vez circular, pero al final el nacionalismo se mantiene como el compromiso principal, encima de cualquiera ideología de la lucha de clases. Sobre esto, Zavaleta (2011c) no permite dudas: "No somos anticapitalistas porque nos irrite en sí el que se llama 'modo americano de la vida', ni socialistas porque haya socialismo en la Unión Soviética o en la China o en Cuba. Lo somos compañeros, porque la existencia nacional no puede realizarse sino dentro de un socialismo latinoamericano” (p. 78).

Se ve entonces que todo dentro de esta problemática del joven Zavaleta se compone en torno a la idea de una nación "en sí" que debe hacerse una "para sí" (Zavaleta, 2011a). La crisis del gobierno del MNR en los principios de los 60 hizo que Zavaleta considerara los medios para lograr tal transformación, y que se enfocara en la cuestión de las clases dentro del movimiento nacionalista, pero la meta política fundamental y la concepción de la nación como unidad básica del análisis histórico persistieron en él como ideas heredadas de la generación nacionalista de Montenegro. Sin embargo, en Noviembre 1964 todo cambió. La crisis se volvió golpe de estado hecho por General René Barrientos, y se acabó el gobierno del MNR. Como otros en el partido, Zavaleta terminó huyendo al exilio. El fracaso del proyecto emenerrista sería el propulsor de una transformación teórica y política más profunda que Zavaleta perseguiría por toda la vida.

\section{Lo nacional-popular y las tendencias capitalistas}

Suele decirse que la fase nacionalista inicial de Zavaleta terminó con un giro hacia el marxismo "ortodoxo" durante su exilio, fase que a su vez cedió a otro período más "heterodoxo" y creativo a los fines de los 70. No obstante, la preocupación con la nación como categoría persiste a lo largo de su carrera, hecho evidenciado por la obra Lo nacional-popular en Bolivia, que sirve como culminación de la fase "heterodoxa” aunque no se terminó durante su vida. Se publicó en forma parcial sólo después de su muerte en 1984.

Aquí, cabe investigar cómo la concepción de la nación en esta obra final se puede diferenciar de la que utiliza Zavaleta en su período más temprano que acabamos de analizar. Según Giller (2015), el tiempo que transcurrió entre estas dos épocas se caracteriza por un cambio de énfasis desde una consideración general de las condiciones exteriores de la carencia nacional de Bolivia, o sea la relación colonial o imperial, hacia un compromiso sostenido en la búsqueda de sus dinámicas internas, sobre todo con respecto al estado y las relaciones de clase. Antezana (1991) y Tapia (2002), por su parte, se concentran en una vuelta epistemológica que lleva Zavaleta a unas novedades conceptuales sofisticadas a través de lo que Tapia llama la producción del conocimiento local. Lo que espero hacer aquí es sólo extraer todas las implicaciones de estos cambios para la relación entre el concepto de clase y de la nación. Sin embargo, es una relación cuya reformulación será sintomática de una ruptura más amplia en la problemática de Zavaleta. Al investigar el concepto de clase y las condiciones de su composición bajo el capitalismo, Zavaleta ofrecerá una manera de pensar más allá, pero no a expensas, de la nación.

Aunque el texto de Lo nacional-popular corre en paralelo al Nacionalismo y coloniaje de Montenegro, trazando varios episodios importantes del pasado boliviano, Zavaleta (2008) marca la distancia de su orientación con el rechazo de lo que él llama un "cierto maniqueísmo" de investigaciones históricas que no podrían explicar las varias "formas de articulación” dentro de la historia (p. 13). Para él, ya es insuficiente hablar de una lucha entre la potencia nacional y las cosas que la repriman desde afuera. "Lo que interesa en cambio, a nuestro entender, es advertir el desarrollo contradictorio de los factores, o sea, como si los hombres propusieran algo y los hechos los llevaran indefectiblemente a otro lugar," escribe Zavaleta (2008: 13-14). Lo nacional-popular se construye en torno a tales momentos, los que él nombra como momentos constitutivos, cuando la potencia sólo se hace visible por los contornos de su existencia material. La posibilidad o no posibilidad de la forma nación se lee en cada 
momento por este tipo de análisis específico de las condiciones.

Para entender mejor cómo Zavaleta analiza estos momentos, y así cómo conceptualiza la nación, conviene pensar la problemática de Zavaleta en términos de dos registros epistemológicos, dos niveles de pensamiento cuyos conceptos operan de maneras distintas, que aparecen en Lo nacional-popular y los otros textos maduros. El primero es el registro de la totalización/descomposición, y el segundo es el de la particularización/composición. Ya se justificarán estas calificaciones.

El registro de la totalización/descomposición es el nivel de investigación permitido por la comprensión de Marx que la historia del capitalismo implica una compresión de tiempo y una densidad aumentada del espacio social. La transformación histórica puede pasar en el capitalismo mucho más rápido que en otro momento histórico y los efectos de cualquier evento son ampliados. Esta característica del capitalismo, según la lectura de Zavaleta (2008), crea la posibilidad de una transformación total, es decir revolucionaria. Para explicar esta faceta del capitalismo, Zavaleta (2008) propone varios conceptos que se puede entender en este registro, siendo clave el de la democratización social. Cita a Weber para definir esto como el proceso de individuos desatados, jurídicamente libre, con las formas de igualdad civil y política que posibilitan la democracia formal. Se complementa esta idea con las de Marx sobre el estado de separación en el cual la ruptura de las relaciones pre-capitalistas funda las condiciones del obrero doblemente libre y la generalización, al menos posible, del trabajo asalariado. Zavaleta nota que el proceso de la democratización social es condición del intersubjetividad, que se califica como "la interacción entre hombres libres que se reconocen entre sí en cuanto tales.” La conexión entre estos varios conceptos se elabora así:

"Pues bien, es a esta interacción generalizada o intersubjetividad, que es la consecuencia de la circulación total sobre los sujetos, a lo que hay que atribuir la construcción de las grandes totalizaciones modernas, desde la clase social (que por eso no se parece a ninguna clase social de otra época) hasta la propia multitud o masa, desde la nación hasta el Estado” (Zavaleta, 2008: 133).

Este proceso de la creación de individuos como sujetos reconocidos jurídicamente forma la base de todas las posibilidades de nuevos modos de unidad política y social dentro del capitalismo, permitiendo la construcción del “continuum mercado-interno-Estado nacional-democracia burguesa, etc.” (Zavaleta, 2013b: 643).

Hay distintas maneras en las cuales se puede interpretar la importancia de este conjunto de conceptos y su relación con la historia concreta. Zavaleta parece a veces sugerir una distinción geográfica-histórica por la cual tales conceptos sólo darían el conocimiento correcto de la historia europea como caso paradigmático de la totalización. En este caso, la presupuesta creación homogénea y efectiva de individuos jurídicamente libre en Europa o tal vez norteamérica sería la regla, permitiendo conocimiento cuantificable, y otros casos desviaciones que eluden tal posibilidad, requiriendo otras herramientas por los efectos permanentes de haber entrado tarde al sistema mundial. Si bien Zavaleta parece pensar así a veces, hay que preguntar si los momentos que la ofrecen están en concordancia o no con sus más penetrantes pensamientos o decisiones metodológicas. Sería extraño, por ejemplo, seguir la tendencia de diferenciar geográficamente el conocimiento hasta el peligro que Hernán Ouviña (2010) nombra como "el exotismo, que absolutizaba la especificidad de América Latina (su cultura, su historia, su estructura social, etc.) acabando por enjuiciar al propio marxismo como doctrina exclusivamente europea” (p. 196). Por más fuerte que sea el enfoque en lo local en las obras de Zavaleta, se sabe que esto no le guio ni al abandono de la teoría marxista, ni a ninguna cesura decidida entre esta teoría y la realidad de América Latina.

Otra manera de interpretar los comentarios sobre el proceso de la totalización es simplemente como el telos del desarrollo histórico bajo el capitalismo. Sea donde sea, este proceso sería el futuro de cualquier sociedad, y su cumplimiento haría posible una transformación total hacia el socialismo. Pero por esta vía, se puede caer en otro “flagelo” que según Ouviña (2010) ha siempre amenazada el pensamiento político en América Latina: “el europeísmo, que tendía a trasladar mecánicamente a esta realidad -y sobre la base de una concepción unilineal de 
la historia- los modelos de desarrollo económico y social occidentales en su ‘evolución’ histórica....” (p. 196-7). En otras palabras, la falacia sería pensar no tanto en términos de la diferencia esencial, sino en términos que implican que los periféricos no europeos sean atrasados o subdesarrollados.

La distinción que propongo entre los dos registros epistemológicos no tiene ninguna correspondencia a la división Este/Oeste o Norte/Sur por un lado, ni con la presuposición de un futuro lineal o pre-determinado para el capitalismo por el otro. Si Zavaleta escribe de Marx y Weber en el primer registro de totalización, es porque estos dos pensadores observaron algo nuevo en el capitalismo, pero no por eso necesariamente universal en sus efectos. Lo que ellos notaron es una tendencia: una orientación histórica impulsada por las relaciones sociales capitalistas pero no siempre presentes en su actualidad, y por eso no considerado como paradigma de la realidad capitalista. Pero en contra de lo que se puede esperar, esta tendencia no se trata tanto de la construcción de las condiciones modernas mismas, sino de la condición negativa que posibilita esta construcción, la existencias de individuos atomizados. En otras palabras, la tendencia subyacente en los conceptos de totalización puede pensarse como la descomposición de las formas sociales y estructuras de clase más antiguas que libera los individuos para que se vuelvan portadores de las relaciones de producción capitalistas.

Si se entiende esto, se puede reconocer que el segundo registro en el pensamiento de Zavaleta es precisamente $-\mathrm{y}$ siempre tiene que existir una- la tendencia opuesta: el registro de la composición y particularidad. Esto se refiere a la re-articulación de nuevas posibilidades y formas, nuevas colectividades y relaciones sociales que surjan con el capitalismo y sus clases modernas. Clarifiquemos la diferencia: la descomposición/totalización refiere esencialmente a un proceso de abstracción, el distanciarse de las circunstancias individuales hacia la igualdad jurídica del mercado, la producción del valor, y la ciudadanía. Es un proceso que se desarrolla con la introducción del capitalismo en cualquier lugar. Por contraste, el registro de la composición llama la atención a la especificidad histórica, la consideración local que fue al centro de las investigaciones de Zavaleta en los años 70 y 80 . No obstante, la diferencia analítica no debe oscurecer que estamos tratando de pensar dos facetas simultáneas e inseparables de la misma historia; la totalización no existe en forma pura ni en Europa ni en el porvenir del capitalismo. Sino, siempre tiene lugar como tendencia entre contenidos locales que condicionan la particularidad y la recomposición que des-atomiza y des-individualiza a los que están separados de los medios de producción.

Ya podemos alumbrar la importancia de esta perspectiva dual para la cuestión del sujeto político y los conceptos de clase y nación en Lo nacional-popular. En un movimiento teórico revelador de la ambigüedad que ya se ha notado aquí, Zavaleta (2008) introduce la discusión del concepto de la nación con una comparación histórica entre los eventos de la guerra federal boliviana y los procesos de unificación nacional italianos y alemanes del siglo

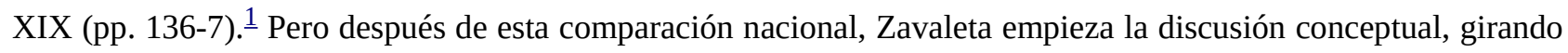
desde la aceptación de la nacionalidad europea como referente empírica del concepto -que requiere pensarse Bolivia como contraejemplo- hacia una examinación misma de la categoría misma de la nación. Cita la famosa definición de Stalin (2002): "Nación es una comunidad humana estable, históricamente formada y surgida sobre la base de la comunidad de idioma, de territorio, de vida económica y de psicología, manifestada ésta en la comunidad de cultura." Entonces, analiza los varios componentes de esta definición en el caso de Bolivia. Después de haber tratado cada uno, pronuncia su juicio sobre sus pesos relativos:

"Con toda la importancia que pudieran tener los argumentos raciales, espaciales y lingüísticos, lo que Stalin llamó problemas de ‘vida económica’ y de ‘psicología’ o comunidad de cultura son sin duda los que tiene un valor más concluyente, aunque no tendrían sino un significado relativo si no los remitiéramos a su fase originaria, esto es, a la discusión del momento constitutivo" (Zavaleta, 2008: 140; cursivas añadidas).

El concepto formal de la nación así se refiere hacia una idea de convergencia histórica que se representa por el concepto del momento constitutivo. Podemos decir que este es el concepto precisamente de la articulación de descomposición y composición en su relación mutua. La creación del "individuo jurídicamente libre, que es una suerte de ciudadanía económica,” postula una relación entre la fuerza de trabajo y el capital:

“Esta ‘vida económica en común’ puede ocurrir, por tanto, con un mayor grado de participación 
desde el individuo desprendido o como un hecho que le sobreviene. Es demasiado evidente que el grado de consenso con que suceda la subsunción formal le da una connotación distinta a cada pacto constitutivo. Es tal interacción generalizada la que produce la sustancia social o materia nacional que lo mismo puede llamarse valor y es en lo que radica la base material de lo nacional en el modo de producción capitalista” (Zavaleta, 2008: 141).

La posibilidad misma de nacionalizarse entonces se funda en un momento de descomposición que es también el momento de la constitución de las relaciones de clase capitalistas (la producción del valor), lo cual a su vez puede manifestarse como cuestión de poder relativo de cada clase (el grado de consenso), i.e., su composición. Por lo tanto, mientras la antigua problemática tomó la potencia constituyente como algo ya nacional, y vio las clases sociales en términos de sus intereses vis-a-vis la meta de la organización de la nación, aquí se ve que lo constituyente es también un momento de contingencia. Las clases son participantes colectivos en formación dentro de en un proceso cuyo finalidad no se sabe, y que, aunque posibilita la nación, puede producir varios tipos de “aglutinación civil” (Zavaleta, 2008: 132).

Es importante notar aquí que incluso cuando Zavaleta recurre a diferenciar entre el occidente y el resto del mundo, o los países capitalistas avanzados y los atrasados, tiende a reconocer que la dialéctica descomposición/composición del momento constitutivo implica una variación radical. Aun las formaciones sociales europeas se encontraron con condiciones históricas específicas -sus propias “formas primordiales”- en las cuales emergieron las relaciones capitalistas, por adentro o desde afuera. Por tanto, lo que a veces se considera como tipo ideal europeo de formación nacional también se ve en otros momentos como tipo de fantasía o idealización:

\begin{abstract}
"Nacional es reconocerse todos como lo mismo, en alguna medida y en cierto hábitat. Ésta sería, con todo, una versión eglógica de la nacionalización, que suele ser un acontecimiento mucho más imperativo y autoritario. Parecería en verdad un proceso lógico que los hombres actúen entre sí y produzcan algo común a todos ellos, pero a la vez no parecido específicamente a ninguno de ellos. Esto es lo que más se aproxima a la revolución democrática entendida como revolución nacional. Es un tipo de lucubración que tiene algo de los quimeristas. En los hechos, la revolución pasiva ha existido, la vía junker ha existido y ha existido sin duda la nacionalización reaccionaria o nacionalización forzosa....” (Zavaleta, 2008: 102).
\end{abstract}

La especificidad del momento constitutivo se describe entonces a través de las relaciones entre las clases y además sus historias y composiciones internas. La manera en que los individuos llegan a un estado de disponibilidad, término que Zavaleta utiliza para decir abiertos a novedades ideológicas y políticas, tiene efectos en el proceso constitutivo, o por formas verticales -como el uso de la riqueza por una clase sobre otra, el desplazamiento forzado de la tierra, etc.- o por formas horizontales como la revolución. Zavaleta (2008) lo resume así: “Incluso si la intersubjetividad existe in abstracto, deberá verse cuál es el camino que se ha seguido para llegar a ella porque

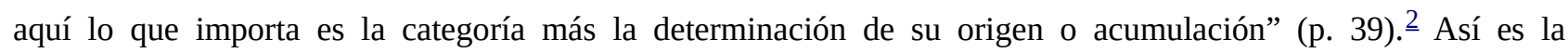
perspicacia de los dos registros conceptuales que siempre apunta a la especificidad de cada caso.

Todo esto nos lleva a la conclusión que así como se puede pensar el momento constitutivo como el momento nacional por excelencia, es también siempre el momento no nacional. ¿Qué quiere decir esto? El espacio social abstracto de la formación de la nación es inseparable de la realidad concreta que lo hace desnivelado, con contornos indistintos, y forma no estable. El momento constitutivo, aunque común a toda nación, es el momento cuando se pierde la apariencia de nación, tanto en la actualidad como en la vista del historiador. Las relaciones que tal vez congelan como nación se revelan como algo distinto, y la imposibilidad permanente de su clausura se ve claramente debido a su conflictualidad. La persistencia de esta conflictualidad interna, al lado de su aglutinamiento, es lo que permite que tales momentos sean calificados como constitutivos. Esta postulación es coherente con lo que a menudo se entiende como la gran intervención de Zavaleta. Como explica Elvira Bórquez Concheiro (2006): 
“De alguna forma, la contribución central de René Zavaleta está en este llamado de atención permanente sobre aquello que no entra en esquema alguno, que tiene, por tanto, un carácter no rutinario. Por ello, su obra nos conduce hacia el estudio de las paradojas sociales, del desarrollo contradictorio, de las conductas inesperadas de los actores sociales y, desde luego, de las catástrofes sociales” (p. 187).

Si la noción de democratización social o totalización es en alguna manera el esquema abstracto de una tendencia, el momento constitutivo apunta a las particularidades que eluden la reducción a ella. La nación constituida es siempre una nación no idéntica a sí misma, una nación con excesos, y la figura de sus excesos siempre tiene algo de la lucha entre clases.

\section{Abigarramiento y totalización bajo el neoliberalismo}

Las dinámicas del capitalismo neoliberal desde los años 70 han experimentado una rearticulación de las dos tendencias que se han identificado aquí como totalización/descomposición y particularización/composición. El resultado es una versión ideológicamente distinta (neoliberal) de lo que Zavaleta ha llamado la democratización social: un esfuerzo por generalizar las relaciones de mercado, a privatizar tanto lo público como lo común, a colocar los medios de bienestar en el dominio de la llamada sociedad civil, a romper formas de colectividad que pesaba sobre la llamada eficacia, y a permitir que el capital financiero sea cada vez más móvil. En un sentido, todo implica una suerte de descomposición que alcanza nuevos niveles de abstracción de los individuos, con el intento por la clase dominante de aplanar el espacio global para los flujos del capital y expandir las relaciones de producción capitalistas. Pero al contrario a la concepción de Zavaleta, esta tendencia no hizo el trabajo preparatorio de la expansión o fortalecimiento de la nación, al menos a corto plazo. La llamada globalización creó una nueva "universalidad real,” con el efecto de vincularse la mayor proporción de individuos en la historia del mundo por el movimiento de mercancías, pero simultáneamente va rompiendo la estabilidad de las categorías de la vida de masas que había significado la “universalidad ideal” en el siglo XX (Balibar, 2002). Sin embargo, si pensamos a Zavaleta no tanto como teórico de la nación, sino el de los límites o aun la falta de la nación, y también de la composición de clase, su pensamiento parece totalmente contemporáneo a nuestra realidad.

La ruptura que ocurre en las relaciones conceptuales en el pensamiento maduro de Zavaleta no es un simple desplazamiento, como si las categorías nación y clase quedaran esencialmente igual que antes, pero con una diferente relación mutua. Sino que es sintomático de un cambio total de problemática; los dos conceptos, por cambiar cómo relacionarse, se cargan con nuevas significancias. El cambio es además el fundamento de la reorientación explícitamente epistemológica que hay que tomar en cuenta para abordar las nuevas realidades del capitalismo: la noción del proletariado como sujeto colectivo que hace posible el conocimiento moderno. Esta clase se considera como el fragmento de modernidad cuya "horizonte de visibilidad" puede explotarse -y fue explotado por Marx- para la producción del conocimiento social dentro de la sociedad marcada por el capitalismo (Zavaleta, 2013b: 646). Este sujeto no puede considerarse en abstracto. Antezana (1991) correctamente lee en Zavaleta la fórmula de clase como “colocación estructural” + "historia concreta”, la cual reconoce el rol del proletariado como explotado en la producción capitalista, pero sin atribuirle intereses reduccionistas o economicistas, ya que estos surgen de la fórmula por su “articulación interna,” o sea su composición (p. 11). Como lo pone Zavaleta (2013b), concretando el vínculo entre la composición de la clase obrera y la producción del conocimiento: "La destrucción de su ser individual es la condición para que aparezca el horizonte de visibilidad general y por eso, la ciencia que se produce a partir del uso de dicho horizonte es también el único rescate de los hombres en su nuevo ser, que es su ser colectivo” (p. 653). La primera pregunta para analizar el capitalismo hoy en día es por lo tanto ¿cómo es el horizonte del proletariado en sus momentos de recomposición colectiva bajo el neoliberalismo?

Si el proletariado, así concebido, es el sujeto epistemológico y político dentro de la problemática madura de Zavaleta, luego ¿cuál es su objeto? En El poder dual, Zavaleta adopta el término formación económico-social, hablando de la articulación de los modos de producción, y así colocando su intervención en aquella debate global 
sobre el asunto de los años 1970s (Antezana 1991; Giller 2015). Para Lo nacional-popular y otros escritos del mismo periodo, Zavaleta empieza a escribir sobre las especificidades del concepto de sociedad abigarrada, término que encapsula los resultados heterogéneos y rugosos del proceso constitutivo de descomposición/composición. Antezana (1991) asevera que la importancia de este concepto es que enfatiza la calificación mutua de las varias partes componentes en una formación heterogénea e implica que cada parte pierde su esencia previa por así calificarse. Tapia (2002) escribe que el concepto de sociedad abigarrada "sirve para pensar no sólo la diversidad sino sobre todo lo problemático de su coexistencia social; y también lo problemático del conocimiento de una de las partes por otras y del conocimiento de cada una de ellas” (p. 431). En un momento cuando se hacen cada vez más densas las redes de relaciones sociales mundiales por el aumento de flujos tanto de capital como de la fuerza de trabajo, y cuando estos flujos y las formas colectivas que crean ponen en duda la fuerza de lo nacional, la sociedad abigarrada puede pensarse como el concepto de esta duda. Por eso, vale considerar su importancia aun afuera de los contextos estrictamente postcoloniales.

Sería equivocado, por razones bastante obvias, sugerir que vivimos en un mundo posnacional, pero al mismo tiempo, cualquier nuevo nacionalismo -o de apariencia más progresista como en América Latina, o más reaccionario como en los EEUU y Europa- solo puede entenderse tomando en cuenta que su rearticulación sigue un periodo de descomposición fuerte. Más allá del nivel descriptivo que reconoce una diversidad creciente de la vida social, las formas particulares de esta diversidad tienen que conocerse. Y para alcanzar este conocimiento, se necesita más investigación al lado de la recomposición de sujetos de las clases subalternas para trazar y entender sus horizontes de visibilidad, especialmente más allá de las ideologías de la nación. Si seguimos a donde nos apunta la obra más sofisticada de Zavaleta, encontramos la contingencia de la nación como formación política. En lugar de lo nacional-popular, se puede buscar lo que Raquel Gutiérrez Aguilar (2008) llama lo comunitario popular, que designa para ella en el caso de Bolivia las nuevas colectividades que no caben dentro de nacionalismo reconstituido. En cualquier sitio esta idea podría incluir a los sujetos colectivos subnacionales, como comunidades indígenas, y también los migrantes y refugiados, cuyas nuevas conexiones suelen trasladar formas comunales desde lugares rurales hasta megaciudades, a menudo cruzando las fronteras nacionales. Además, se ve la construcción de lo común a veces en los nuevos lazos transnacionales de producción incluyendo flujos de bienes y recursos y reformando las relaciones, por ejemplo, entre trabajadores, cuentapropistas mercantiles, y cadenas de suministro en las economías populares. Todo esto puede servir como punta de partida para entender las composiciones y horizontes de la clase obrera, en sí abigarrada, bajo o después del neoliberalismo. Al fin de cuentas, lo que Zavaleta nos enseña es que aun cuando el nacionalismo está sobre la agenda, la lucha de clase presenta otras posibilidades inesperadas. Para Zavaleta esto fue tal vez una realización trágica. Para nosotros, tiene la potencia de ser una salida.

\section{Notas}

1 Así se presenta el impulso de utilizar los eventos de Europa como modelos empíricos de los conceptos históricos, dando credibilidad a la idea que el eje epistemológico de Zavaleta en este texto sea esencialmente geográfico. Los lazos de esta concepción con la de la problemática nacionalista revolucionaria, donde se supone que la diferencia nacional siempre estructura la historia, quedan bastante claros.

$\underline{2}$ Aun cuando Zavaleta (2008) da a Inglaterra como “paradigma” de la creación del estado de separación, él nota que no es el único ejemplo, y que entre las naciones europeas, Francia se presenta como contrapunto radicalmente distinto (p. 144).

\section{Bibliografía citada}

Antezana, L. (1991). Dos conceptos en la obra de René Zavaleta Mercado, College Park, University of Maryland. 
Badiou, A. (2007). The century, London, Polity.

Balibar, E. (2002). Politics and the other scene, London, Verso.

Concheiro Bórquez, E. (2006). “René Zavaleta: Una mirada comprometida”, en M. Aguiluz Ibargüen y N. Rios Méndez (Eds.). René Zavaleta Mercado: Ensayos, testimonios y re-visiones, Buenos Aires, Miño y Dávila, pp. $179-188$

Dunkerley, James (2007). “The origins of the Bolivian Revolution in 1952: Some reflections”, en Bolivia: Revolution and the power of history in the present, London, Institute for the Study of the Americas, pp. 214-254

Giller, D. M. (2015). “René Zavaleta Mercado frente a la ‘Teoría de la dependencia’: Algunas Cuestiones en torno de la noción de la determinación dependiente y la forma primordial”, en Revista Intersticios de la política y la cultura, 4 (8), pp. 115-132.

Gutiérrez Aguilar, R. (2008). Los ritmos de Pachakuti: movilización y levantamiento popular-indígena en Bolivia (2000-2005), Buenos Aires, Tinta Limón.

Mansilla, H.C.F. (2015). Una mirada crítica sobre la obra de René Zavaleta Mercado, La Paz, Rincón Ediciones.

Montenegro, C. (1967). Nacionalismo y coloniaje, Buenos Aires, Pleamar. (Trabajo originalmente publicado 1944).

Ouviña, H. (2010). “Traducción y nacionalización del Marxismo en América Latina: Un acercamiento al pensamiento político de René Zavaleta Mercado”, en OSAL, Buenos Aires- CLACSO, 11(28), pp. $193-207$.

Stalin, J. (2002). Marxismo y la cuestión nacional. Recuperado de: https://www.marxists.org/espanol/stalin/1910s/vie1913.htm. (Trabajo originalmente publicado 1913)

Tapia, Luis (2002). La producción del conocimiento local: Historia y política en la obra de René Zavaleta, La Paz, Muela del Diablo.

Zavaleta Mercado, R. (2008). Lo nacional-popular en Bolivia, La Paz, Plural. (Trabajo originalmente publicado 1986)

Zavaleta Mercado, R. (2011a). “Bolivia: El desarrollo de la conciencia nacional”, en M. Souza Crespo (Ed.). René Zavaleta Mercado Obra completa I, La Paz, Plural (Trabajo originalmente publicado 1967) pp. 121-201

Zavaleta Mercado, R. (2011b). “Cinco años de Revolución Nacional en Bolivia”, en M. Souza Crespo (Ed.). René Zavaleta Mercado Obra completa I, La Paz, Plural (Trabajo originalmente publicado 1957), pp. 529-534

Zavaleta Mercado, R. (2011c). “Estado Nacional o pueblo de pastores”, En M. Souza Crespo (Ed.), René Zavaleta Mercado Obra completa I, La Paz, Plural (Trabajo originalmente publicado 1963), pp. 57-95

Zavaleta Mercado, R. (2011d). “La Revolución Boliviana y el doble poder”, en M. Souza Crespo (Ed.), René Zavaleta Mercado Obra completa I, La Paz, Plural (Trabajo originalmente publicado 1962), pp. 535-543

Zavaleta Mercado, R. (2013a). “Las masas en noviembre”, en M. Souza Crespo (Ed.). René Zavaleta Mercado Obra completa II, La Paz, Plural. (Trabajo originalmente publicado 1983), pp. 97-142

Zavaleta Mercado, R. (2013b). “Problemas de la cultura”, en M. Souza Crespo (Ed.). René Zavaleta Mercado Obra completa II, La Paz, Plural (Trabajo originalmente publicado 1984), pp. 641-654 